\title{
Special Issue on Intelligent Processing on Image and Optical Information
}

\author{
Seokwon Yeom \\ School of Information and Convergence Technology, Daegu University, Gyeongsan 38453, Korea; \\ yeom@daegu.ac.kr
}

Received: 14 May 2020; Accepted: 25 May 2020; Published: 5 June 2020

check for updates

\section{Introduction}

Intelligent image and optical information processing have paved the way for the recent epoch of new intelligence and information era. Certainly, information acquired by various imaging techniques is of tremendous value, thus, an intelligent analysis of them is necessary to make the best use of it.

The objectives of intelligent processing range from the refinement of raw data to the symbolic representation and visualization of the real world. The extraction and manipulation of the descriptive features are essential for such a task [1]. It comes through unsupervised or supervised learning based on statistical and mathematical models or computational algorithms. With recent advances in computing power and learning algorithms, many applications have become more practical and further development is expected.

This Special Issue focuses on the intelligent processing of images and optical information acquired by various imaging methods. Images are commonly formed via visible light; there are other imaging sources that represent scenes and objects in a multi-dimensional frame [2]. For example, radiography can be used to see internal objects for medical diagnosis and non-destructive inspection. Infrared imaging detects thermal variations in a non-visible environment. Spectral imaging is useful for collecting information at high spectral resolution or over a wide spectrum. Microscopic imaging reproduces the microscopic world, while satellite imaging probes the Earth from space. Since the statistical distribution depends on the nature of the source, suitable intelligent processing should be contrived accordingly. A broad range of research fields is included in the Special Issue. Many studies focus on object classification and detection. Registration, segmentation, and fusion are performed between a series of images. Many valuable and up-to-most recent technologies are provided to solve the real problems in selected papers.

A total of 61 manuscripts were submitted and only 18 research, and one review, papers were verified through a thorough review process. The first volume of the Special Issue on the topic is closed; more in-depth research of the same topic is expected in the second volume of the Special Issue. It is anticipated that the scope of the intelligent processing would be even broader in the future.

\section{Intelligent Processing on Image and Optical Information}

This Special Issue was introduced to collect the latest research on relevant topics, and more importantly, to address the current practical and theoretical challenges. In the following, the papers are categorized into several subtopics; classification and detection, feature extraction and segmentation; estimation and localization; registration and fusion; compression, completion, and correction; optimization and clustering.

\subsection{Classification and Detection}

In the first paper of this category, entitled 'Change Detection of Water Resources via Remote Sensing: An L-V-NSCT Approach', Wang Xin, Tang Can, Wang Wei, and Li Ji [3] presented landscape 
monitoring via satellite remote sensing. The authors analyzed the texture features of the image to address the characteristics of changes in certain types of surface, such as rivers and lakes.

Texture classification is an important topic for many applications in image analysis. A paper, entitled 'A Texture Classification Approach Based on the Integrated Optimization for Parameters and Features of Gabor Filter via Hybrid Ant Lion Optimizer' by Mingwei Wang, Lang Gao, Xiaohui Huang, Ying Jiang, and Xianjun Gao [4], proposed a new texture classification based on the integrated optimization of the parameters and features of the Gabor filter.

Calcaneal fractures are often caused by accidents during exercise or activity. In general, the detection of calcaneal fractures is still performed manually through computer tomography (CT) image observation. Wahyu Rahmaniar and Wen-June Wang [5], in their paper, 'Real-Time Automated Segmentation and Classification of Calcaneal Fractures in CT Images' proposed a method for detecting calcaneal fractures through localization and color segmentation of calcaneus.

Two papers on biological research, entitled 'Automatic Zebrafish Egg Phenotype Recognition from Bright-Field Microscopic Images Using Deep Convolutional Neural Network' [6] and 'Zebrafish Larvae Phenotype Classification from Bright-field Microscopic Images Using a Two-Tier Deep-Learning Pipeline' [7] are presented for zebrafish egg recognition. In the former paper, Shang, Ling Long, Sijie Lin, and Fengyu Cong addressed an automated zebrafish egg microscopic image analysis based on deep convolution neural networks (CNN). This study applies deep learning technique to classify fertilized and unfertilized zebrafish eggs from bright-field microscopic images. Transfer learning and data augmentation schemes were used to overcome the problem of small unbalanced training datasets. In the latter paper, Sijie Lin and his coworkers proposed a deep-learning pipeline for zebrafish larvae phenotype classification from brightfield microscopic images. Facing the difficulties of scarce training data, they used a two-tier classification pipeline.

As the deep-learning model develops, a huge number of images is required for training. Jeong gi Kwak and Hanseok Ko [8] described in their article: 'Unsupervised Generation and Synthesis of Facial Images via an Auto-Encoder-Based Deep Generative Adversarial Network (GAN)' an auto-encoder-based GAN with an enhanced network structure and training scheme for database augmentation and image synthesis.

As fruit production worldwide increases, the use of spectroscopy to monitor the surface condition of food continues to increase. A paper entitled 'Detecting Green Mold Pathogens on Lemons Using Hyperspectral Images' by Yuriy Vashpanov, Gwanghee Heo, Yongsuk Kim, Tetiana Venkel, and Jung-Young Son [9] investigates spectral images to detect green mold pathogens that parasitize on the lemon surface.

In their review paper entitled 'Computer Aided Weld Defect Detection from Radiography Images', Wenhui Hou, Dashan Zhang, Ye Wei, Jie Guo, and Xiaolong Zhang [10] reviewed the automatic defect inspection in three aspects: pre-processing, defect segmentation and defect classification. The achievement and limitations of classification based on feature extraction, selection and classifier are summarized and the application of new models based on deep learning were introduced.

\subsection{Feature Extraction and Segmentation}

The paper entitled 'Feature Extraction with Discrete Non-Separable Shearlet Transform and Its Application to Surface Inspection of Continuous Casting Slabs' by Xiaoming Liu, Ke Xu, Peng Zhou, and Huajie Liu [11] proposes a new feature extraction technique for the direction and texture information of surface defects of continuous casting slabs with complex backgrounds.

Wending Liu, Hanxing Liu, Yuan Wang, Xiaorui Zheng, and Junguo Zhang [12], in their paper on 'A Novel Extraction Method for Wildlife Monitoring Images with Wireless Multimedia Sensor Networks (WMSNs)', proposed an segmentation method with a low computational complexity, which can extract the target area from the background based on the image texture and color information. This proposed method can realize more accurate extraction of wildlife monitoring images and effectively support image transmission in wireless sensor networks. 


\subsection{Estimation and Localization}

For autonomous driving, it is important to obtain precise and high-frequency localization of the vehicle. The inertial measurement unit (IMU), wheel encoder, and lidar odometry are utilized together to estimate the ego-motion of the unmanned ground vehicle in the paper entitled 'IMU-Aided High-Frequency Lidar Odometry for Autonomous Driving' by Hanzhang Xue, Hao Fu, and Bin Dai [13].

Dough fermentation plays an essential role in the bread production process, and its success is critical to producing high-quality products. In the paper entitled 'Determination of the Optimal State of Dough Fermentation in Bread Production by Using Optical Sensors and Deep Learning', Lino Antoni Giefer, Michael Lütjen, Ann-Kathrin Rohde, and Michael Freitag [14] proposed a novel method for the continuous monitoring of the volumetric parameters of dough piece during the fermentation process.

\subsection{Registration and Fusion}

In the paper entitled 'Multi-Sensor Face Registration Based on Global and Local Structures', Wei Li 1, Mingli Dong, Naiguang Lu, Xiaoping Lou, and Wanyong Zhou [15] introduced a novel multi-sensor face image registration method. This work utilizes global geometrical relationships and local shape features to register visible and infrared (IR) facial images for fusion-based recognition.

The paper entitled 'Multifocus Image Fusion Using a Sparse and Low-Rank Matrix Decomposition for Aviator's Night Vision Goggle' by Bo-Lin Jian, Wen-Lin Chu, Yu-Chung Li, and Her-Terng Yau [16] proposed an autofocusing and image fusion algorithm with sparse and low-rank matrix decomposition, to inspect the night vision goggles (NVG) of the aircraft. Their method can solve the multi-focusing problem caused by the mechanism error of the NVG.

\subsection{Compression, Completion, and Correction}

The paper entitled 'Error Resilience for Block Compressed Sensing with Multiple-Channel Transmission' by Hsiang-Cheh Huang, Po-Liang Chen, and Feng-Cheng Chang [17] proposed an error resilient transmission scheme for block compressed sensing. In their paper, the compressed information is transmitted over the multiple independent and lossy channels. By introducing correlation to multiple description coding, errors induced in the lossy channel are effectively alleviated.

The incomplete images can contain many missing pixels distributed over the entire image. In the paper entitled 'Image Completion with Hybrid Interpolation in Tensor Representation', Rafał Zdunek and Tomasz Sadowski [18] proposes an interpolation algorithm for a wide spectrum of image-completion problems. Their algorithm outperforms other conventional methods with a considerably shorter computational runtime.

In the paper entitled 'A Correction Method for HeatWave Distortion in Digital Image Correlation Measurements Based on Background-Oriented Schlieren', Chang Ma, Zhoumo Zeng, Hui Zhang, and Xiaobo Rui [19] proposed a correction method based on the background-oriented schlieren technique. The method can correct the distortion caused by heat waves in measuring image correlation.

\subsection{Optimization and Clustering}

The theoretical aspects of optimization and clustering are emphasized in the last two papers. On the paper entitled 'An Effective Optimization Method for Machine Learning Based on ADAM', Dokkyun Yi, Jaehyun Ahn, and Sangmin Ji [20] proposed a novel optimization method for non-convex cost functions to make machine learning more efficient. The proposed method solves the problem of falling to a local minimum by adding a cost function in the parameter update rule of the adaptive moment estimation (ADAM) method.

The paper entitled 'Boundary Matching and Interior Connectivity-Based Cluster Validity Analysis' by Qi Li, Shihong Yue, Yaru Wang, Mingliang Ding, Jia Li, and Zeying Wang [21] proposed a novel method to evaluate clustering results by the validity index. Their analysis can be applied to select the optimal clustering parameters including the number of clusters. 
Acknowledgments: This issue would not be possible without the contributions of many outstanding authors. I would like to thanks to all authors who submitted their papers to this issue regardless of the final decision. I sincerely hope that comments and suggestions from the reviewers and the editors helped the authors improve their papers. I also appreciate the dedicated reviewers for their efforts to expertise. Finally, I place on record my gratitude to the professional editorial team of Applied Sciences, and special thanks to Stella Zhang, Assistant Editor from MDPI Branch Office, Beijing.

Conflicts of Interest: The author declares no conflict of interest.

\section{References}

1. Gonzalez, R.C.; Woods, R.E. Digital Image Processing, 4th ed.; Pearson: London, UK, 2018.

2. Hecht, E. Optics, 5th ed.; Pearson: London, UK, 2016.

3. Xin, W.; Can, T.; Wei, W.; Ji, L. Change Detection of Water Resources via Remote Sensing: An L-V-NSCT approach. Appl. Sci. 2019, 9, 1223. [CrossRef]

4. Wang, M.; Gao, L.; Huang, X.; Jiang, Y.; Gao, X. A Texture Classification Approach Based on the Integrated Optimization for Parameters and Features of Gabor Filter via Hybrid Ant Lion Optimizer. Appl. Sci. 2019, 9, 2173. [CrossRef]

5. Rahmaniar, W.; Wang, W. Real-Time Automated Segmentation and Classification of Calcaneal Fractures in CT Images. Appl. Sci. 2019, 9, 3011. [CrossRef]

6. Shang, S.; Long, L.; Lin, S.; Cong, F. Automatic Zebrafish Egg Phenotype Recognition from Bright-Field Microscopic Images Using Deep Convolutional Neural Network. Appl. Sci. 2019, 9, 3362. [CrossRef]

7. Shang, S.; Lin, S.; Cong, F. Zebrafish Larvae Phenotype Classification from Bright-field Microscopic Images Using a Two-Tier Deep-Learning Pipeline. Appl. Sci. 2020, 10, 1247. [CrossRef]

8. Kwak, J.; Ko, H. Unsupervised Generation and Synthesis of Facial Images via an Auto-Encoder-Based Deep Generative Adversarial Network. Appl. Sci. 2020, 10, 1995. [CrossRef]

9. Vashpanov, Y.; Heo, G.; Kim, Y.; Venkel, T.; Son, J.Y. Detecting Green Mold Pathogens on Lemons Using Hyperspectral Images. Appl. Sci. 2020, 10, 1209. [CrossRef]

10. Hou, W.; Zhang, D.; Wei, Y.; Guo, J.; Zhang, X. Review on Computer Aided Weld Defect Detection from Radiography Images. Appl. Sci. 2020, 10, 1878. [CrossRef]

11. Liu, X.; Xu, K.; Zhou, P.; Liu, H. Feature Extraction with Discrete Non-Separable Shearlet Transform and Its Application to Surface Inspection of Continuous Casting Slabs. Appl. Sci. 2019, 9, 4668. [CrossRef]

12. Liu, W.; Liu, H.; Wang, Y.; Zheng, X.; Zhang, J. A Novel Extraction Method for Wildlife Monitoring Images with Wireless Multimedia Sensor Networks (WMSNs). Appl. Sci. 2019, 9, 2276. [CrossRef]

13. Xue, H.; Fu, H.; Dai, B. IMU-Aided High-Frequency Lidar Odometry for Autonomous Driving. Appl. Sci. 2019, 9, 1506. [CrossRef]

14. Giefer, L.A.; Lütjen, M.; Rohde, A.K.; Freitag, M. Determination of the Optimal State of Dough Fermentation in Bread Production by Using Optical Sensors and Deep Learning. Appl. Sci. 2019, 9, 4266. [CrossRef]

15. Li, W.; Dong, M.; Lu, N.; Lou, X.; Zhou, W. Multi-Sensor Face Registration Based on Global and Local Structures. Appl. Sci. 2019, 9, 4623. [CrossRef]

16. Jian, B.L.; Chu, W.L.; Li, Y.C.; Yau, H.T. Multifocus Image Fusion Using a Sparse and Low-Rank Matrix Decomposition for Aviator's Night Vision Goggle. Appl. Sci. 2020, 10, 2178. [CrossRef]

17. Huang, H.C.; Chen, P.L.; Chang, F.C. Error Resilience for Block Compressed Sensing with Multiple-Channel Transmission. Appl. Sci. 2020, 10, 161. [CrossRef]

18. Zdunek, R.; Sadowski, T. Image Completion with Hybrid Interpolation in Tensor Representation. Appl. Sci. 2020, 10, 797. [CrossRef]

19. Ma, C.; Zeng, Z.; Zhang, H.; Rui, X. A Correction Method for Heat Wave Distortion in Digital Image Correlation Measurements Based on Background-Oriented Schlieren. Appl. Sci. 2019, 9, 3851. [CrossRef]

20. Yi, D.; Ahn, J.; Ji, S. An Effective Optimization Method for Machine Learning Based on ADAM. Appl. Sci. 2020, 10, 1073. [CrossRef]

21. Li, Q.; Yue, S.; Wang, Y.; Ding, M.; Li, J.; Wang, Z. Boundary Matching and Interior Connectivity-Based Cluster Validity Analysis. Appl. Sci. 2020, 10, 1337. [CrossRef] 